\title{
Robust time-varying shortest path with arbitrary waiting time at vertices
}

\author{
Gholamhassan Shirdel ${ }^{1, *}$ and Hassan Rezapour ${ }^{1}$ \\ ${ }^{1}$ Department of Mathematics, University of Qom, Qom, Iran \\ E-mail: 〈\{Shirdel81math, Hassan.Rezapour\}@gmail.com〉
}

\begin{abstract}
In this paper, we propose an algorithm for solving the robust time-varying shortest path problem in which the waiting time at any vertex is not restricted. The problem is to find the shortest path connecting source node $s$ and sink node $i$ in a timevarying network flow such that the time of the path is at most $T$, where $T$ is a given integer, the transit costs are uncertain and waiting time is arbitrarily allowed at any of the vertices.
\end{abstract}

Key words: time-varying network, shortest path, robust optimization

Received: June 11, 2016; accepted: August 24, 2017; available online: November 30, 2017

DOI: $10.17535 /$ crorr.2017.0034

\section{Introduction}

Network flows problems (NFP) are important topics in optimization and vital problems in operations research. Static and time-varying classes are considered important branches in NFP. A complete discussion of static NFP has been widely studied by Ahuja et al in [1]. A particular case in time-varying NFP was first introduced by Ford and Fulkerson in [9]. This case was named dynamic NFP and was developed later (see $[2,8,10,12,16]$ ). In [9], Ford and Fulkerson introduced the concept of dynamic flows in networks with the maximal dynamic NFP. In [2], Aronson provided a survey of known results, algorithms and implementations for dynamic NFP models and presented some more general formulations and results for maximum and minimum dynamic NFP. Hoppe focused on an extension of the efficient dynamic network flow algorithms and described the use of parallel mortal edges for a time-varying network flow problem [12]. Moreover, a dynamic NFP was surveyed by Glockner and Nemhauser in [10], with a focus on uncertainty of arc capacities. Fonoberova considered the dynamic NFP with nonlinear cost functions, defined on edges. Moreover, she assumed that the supply and demand functions and capacities of edges also depend on time [8]. Dynamic generative network flow with a timevarying bound was considered by Salehi Fathabadi and Hosseini in [16], in which the flow was dynamically generated at a source node, and dynamically consumed at a sink node and where the arc-flow bounds are time dependent. The general form of time-varying NFP have been discussed in $[4,5,13,14,15,17]$.

${ }^{*}$ Corresponding author. 
Time-dependent delays were treated in the context of shortest path problems by Orda and Rom in [13]. In [5], Cai et al. have developed a technique which solves the time-varying minimum cost flow problem. Nasrabadi and Hashemi dealt with a general minimum cost dynamic NFP in a discrete time model with time-varying transit time and costs, storage costs and capacities and presented a new algorithm (see $[15])$.

Shirdel and Rezapour studied a k-objective time-varying shortest path (TVSP) problem, which cannot be combined into a single overall objective [17]. Moreover, they surveyed the TVSP problem with fuzzy costs and arbitrary waiting time in [18].

\section{Preliminary}

Let $G(V, A, b, c)$ be a time-varying network, where $V$ is the set of vertices and $A$ is the set of arcs. Consider $b(i, j, t)$ and $c(i, j, t)$ as the transit time and the transit cost of an $\operatorname{arc}(i, j) \in A$, respectively, where $t$ is the departure time of the vertex $i$, i.e., the transit time $b(i, j, t)$ is needed to traverse an arc and the transit cost $c(i, j, t)$ is paid for traverse an arc $(i, j)$. Both of them, i.e., $b$ and $c$, depend on the discrete time $t=0,1, \ldots, T$ where $T \geq 0$ is the maximum permissible time to travel from the source vertex $s$ to the target vertex $\rho$. For the general case, we can consider the lower bound $l(i, j, t)$ and upper bound $U(i, j, t)$ for arc $(i, j) \in A$ that are functions of the departure time $t$ at the start vertex $i$ of the $\operatorname{arc}(i, j)$.

The maximum capacity of vertex $i$ for the period of the time $t$ to $t+1$ is denoted by $U(i, t)$, representing the maximum amount of flow that can wait at vertex $i$ during the time period $[t, t+1)$. The waiting cost is defined as $c(i, t)$, the transit cost. $c(i, t)$ is the waiting cost at vertex $i$ for the period of the time $t$ to $t+1$. We also assume that the real waiting time at vertex $i$ is shown by $w(i)$. We presuppose that the waiting time $w(i)$, transit cost $c(i, j, t)$ and the waiting cost $c(i, t)$ are integers, whereas the transit time $b(i, j, t)$ is a non-negative integer. Moreover, let $|A|=m$ and $|V|=n$.

Definition 1 (see [6]). Suppose a path from $i_{1}$ to $i_{k}$ is denoted by $P\left(i_{1}-i_{2}-\cdots-i_{k}\right)$. Let $\alpha\left(i_{1}\right)$ be the arrival time of a vertex $i_{1}$ on $P\left(i_{1}-i_{2}-\cdots-i_{k}\right)$ such that $\alpha\left(i_{1}\right)=$ $t_{1} \geq 0$ and:

$$
\alpha\left(i_{l}\right)=\alpha\left(i_{l-1}\right)+w\left(i_{l-1}\right)+b\left(i_{l-1}, i_{l}, \tau\left(i_{l-1}\right)\right) \quad \text { for } \quad 2 \leq l \leq k,
$$

where $\tau\left(i_{l-1}\right)$ is the departure time of a vertex $i_{l-1}$ for $2 \leq l \leq k$ on $P\left(i_{1}-i_{2}-\cdots-i_{k}\right)$ and we have:

$$
\tau\left(i_{l-1}\right)=\alpha\left(i_{l-1}\right)+w\left(i_{l-1}\right) \quad \text { for } \quad 2 \leq l \leq k .
$$

Meanwhile we let $\alpha(s)=0$ for source vertex $s$.

Definition 2 (see $[6])$. We say that $P\left(i_{1}-i_{2}-\cdots-i_{k}\right)$ is a time-varying path from $i_{1}$ to $i_{k}$, if all $\alpha\left(i_{l}\right), w\left(i_{l}\right)$ and $\tau\left(i_{l}\right)$ for all $1 \leq l \leq k$ on the $P\left(i_{1}-i_{2}-\cdots-i_{k}\right)$ are calculated and specified.

Definition 3 (see [6]). If $P\left(i_{1}-i_{2}-\cdots-i_{k}\right)$ is a time-varying path from $i_{1}$ to $i_{k}$, then: 
I. The time of $P\left(i_{1}-i_{2}-\cdots-i_{k}\right)$ is determined by $\alpha\left(i_{k}\right)+w\left(i_{k}\right)-\alpha\left(i_{1}\right)$.

II. The cost of $P\left(i_{1}-i_{2}-\cdots-i_{k}\right)$ is defined as follows:

$$
\begin{gathered}
\xi(P)=\xi\left(i_{k-1}\right)+c\left(i_{k-1}, i_{k}, \tau\left(i_{k-1}\right)\right)+\sum_{t^{\prime}=0}^{w\left(i_{k}\right)} c\left(i_{k}, t^{\prime}+\alpha\left(i_{k}\right)\right), \\
\text { where, } \xi\left(i_{1}\right)=\sum_{t^{\prime}=0}^{w\left(i_{1}\right)-1} c\left(i_{1}, t^{\prime}+\alpha\left(i_{1}\right)\right) .
\end{gathered}
$$

Definition 4 (see $[6])$. The path $P\left(i_{1}-i_{2}-\cdots-i_{k}\right)$ is a time-varying shortest path from $i_{1}$ to $i_{k}$ within the maximum time period $t$ if for each time-varying path $P^{\prime}$ from $i_{1}$ to $i_{k}$ within the maximum time period $t$, we have:

$$
\xi(P) \leq \xi\left(P^{\prime}\right) .
$$

Definition 5 (see [6]). For a given number $t \leq T$, the cost of shortest path within the time period $t$ is supposed to be $\infty$ if there is no path from $i_{1}$ to $i_{k}$ or the time of all paths be greater than $t$.

\section{Time-varying shortest path with arbitrary waiting time}

Time-varying shortest path problem (T-VSP) requires finding the shortest path $P$ from vertex $s$ as the source vertex to a different vertex $i_{k}$ as the sink, so that the total costs (including transit costs and waiting costs) are minimized, all time constraints are satisfied and the path $P$ has a maximum time of $T$, where $T$ is a specified integer. It is important to mention that, the real applications are constrained due to the waiting times.

It is possible to have shorter time-varying path by considering waiting at a vertex. In other words, $w(i)$ is a decision variable that should be considered in T-VSP. Based on the waiting mode, three cases can be considered. Case 1 is T-VSP with $w(i)=$ $\infty, \forall i \in V$ (waiting times are arbitrary) and the problem is called the time-varying shortest path problem with arbitrary waiting times (T-VSP-AWT). This problem is studied in this section. Case 2 is T-VSP with $w(i)=0, \forall i \in V$ (waiting times are zero for all vertices in $V$ ) and the problem is called the time-varying shortest path problem with zero waiting times and designated as T-VSP-ZWT. Finally, case 3 is T-VSP with $w(i)=u_{i}$ when $u_{i}$ is a finite positive number, each waiting time at a vertex is bounded by a positive number $u_{i}$ and in this case we have the timevarying shortest path problem with bounded waiting times. This problem can also be identified by T-VSP-BWT.

Definition 6. Consider the case T-VSP-AWT. Suppose $d_{A}(j, t)$ is the cost of a time-varying shortest path from $s$ to $j$ over exactly time $t$, where $w(i)=\infty, \forall i \in V$. If there is no such path then let:

$$
d_{A}(j, t)=\infty .
$$


Lemma 1. [6] If $t=0$, then $d_{A}(j, t)=0$ for $j=s$ and $d_{A}(j, t)=\infty$ for $j \neq s$. Moreover if $t>0$, then we have:

$$
d_{A}(j, t)=\min \left\{d_{A}(j, t-1)+c(j, t-1), \min _{(i, j) \in A} \min _{\mathfrak{R}}\left\{d_{A}(i, u)+c(i, j, u)\right\}\right\},
$$

where $\mathfrak{R}=\{u \mid u+b(i, j, u)=t\}$.

Theorem 1. Assign $d_{A^{*}}(j)$ as the cost of a time-varying shortest path from $s$ to $t$ during a maximum time of $T$, where $w(i)=\infty, \forall i \in V$. Then the following holds:

$$
d_{A^{*}}(j)=\min _{0 \leq t \leq T} d_{A}(j, t)
$$

Proof. The theorem is easily proved by definition $d_{A^{*}}(j)$ and lemma 1 .

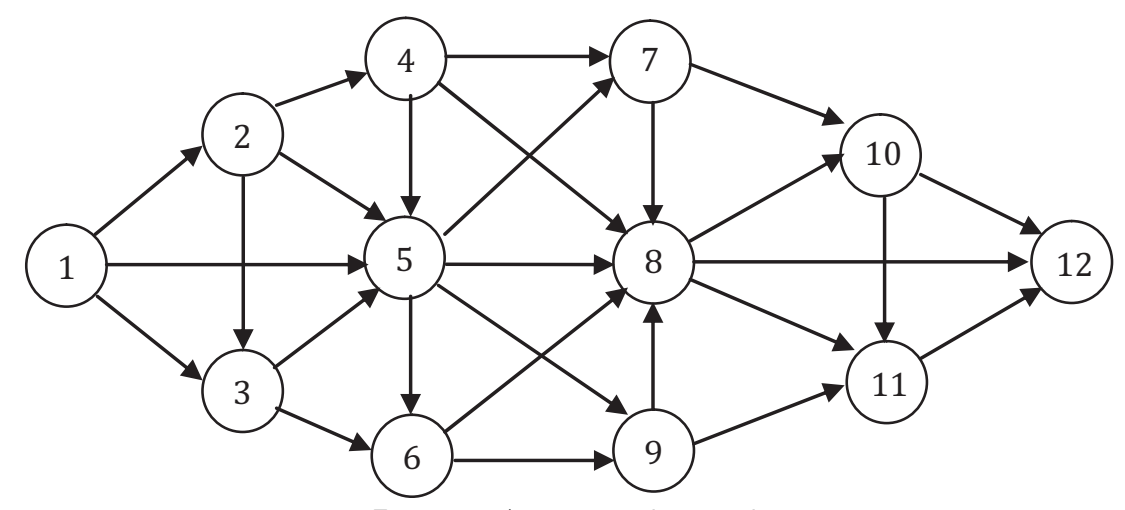

Figure 1: A numerical example

Example 1. Time-varying network $G(V, A, b, c)$ is given in Figure 1, where $T=10$. The purpose is to find the shortest paths from $s$ to another vertex $j$ such that the time of these paths is at most $T=10$. Consider $c(i, t)=0, \forall i \in V, \forall t=0,1, \ldots, 10$.

For $\operatorname{arcs}(2,4),(2,5),(4,7)$ and $(6,8)$, let: $(b(i, j, t), c(i, j, t))=(2,1), \forall t=0,1, \ldots, 10$.

For $\operatorname{arcs}(7,10),(8,12)$ and $(9,11)$, let: $(b(i, j, t), c(i, j, t))=(3,2), \forall t=0,1, \ldots, 10$ and $U(i, j, t)=1, \forall(i, j) \in A, \forall t \in T$.

Moreover, other information about $G$ is provided in Table 1 for Figure 1. We solved T-VSP for 1. By the Theorem 1 and Lemma 1, we have:

$$
\begin{aligned}
& d_{A^{*}}(1)=0, \quad d_{A^{*}}(2)=1, \quad d_{A^{*}}(3)=1, \quad d_{A^{*}}(4)=3, \\
& d_{A^{*}}(5)=1, \quad d_{A^{*}}(6)=4, \quad d_{A^{*}}(7)=3, \quad d_{A^{*}}(8)=3, \\
& d_{A^{*}}(9)=4, \quad d_{A^{*}}(10)=7, \quad d_{A^{*}}(11)=7, \quad d_{A^{*}}(12)=6 .
\end{aligned}
$$




\begin{tabular}{|c|c|c|c|c|c|c|c|c|c|c|c|c|c|c|c|c|c|c|c|c|}
\hline Arc & \multicolumn{2}{|c|}{$(1,2)$} & \multicolumn{2}{|c|}{$(1,3)$} & \multicolumn{2}{|c|}{$(1,5)$} & \multicolumn{2}{|c|}{$(2,3)$} & \multicolumn{2}{|c|}{$(3,5)$} & \multicolumn{2}{|c|}{$(3,6)$} & \multicolumn{2}{|c|}{$(4,5)$} & \multicolumn{2}{|c|}{$(4,8)$} & \multicolumn{2}{|c|}{$(5,6)$} & \multicolumn{2}{|c|}{$(5,7)$} \\
\hline b,c & b & c & b & c & b & c & b & c & b & $\mathrm{c}$ & b & c & b & c & b & c & & c & b & c \\
\hline 0 & 1 & 2 & 2 & 3 & 1 & 1 & 1 & 2 & 2 & 4 & 1 & 3 & 1 & 4 & 1 & 4 & 3 & 2 & 4 & 2 \\
\hline 1 & 1 & 3 & 1 & 3 & 2 & 2 & 1 & 3 & 2 & 3 & 1 & 2 & 2 & 4 & 1 & 4 & 2 & 5 & 2 & 6 \\
\hline 2 & 2 & 4 & 1 & 4 & 1 & 1 & 2 & 4 & 1 & 4 & 2 & 3 & 3 & 5 & 2 & 5 & 1 & 7 & 1 & 7 \\
\hline 3 & 1 & 5 & 1 & 5 & 3 & 4 & 1 & 5 & 1 & 5 & 1 & 6 & 4 & 2 & 3 & 6 & 3 & 6 & 3 & 7 \\
\hline 4 & 3 & 2 & 2 & 4 & 1 & 5 & 3 & 2 & 1 & 6 & 5 & 7 & 5 & 3 & 4 & 2 & 2 & 3 & 1 & 6 \\
\hline 5 & 2 & 3 & 2 & 6 & 2 & 6 & 2 & 3 & 2 & 7 & 6 & 6 & 6 & 7 & 3 & 4 & 1 & 8 & 1 & 4 \\
\hline 6 & 1 & 4 & 1 & 1 & 1 & 8 & 1 & 4 & 1 & 4 & 7 & 4 & 7 & 1 & 2 & 7 & 1 & 3 & 1 & 4 \\
\hline 7 & 2 & 5 & 3 & 3 & 1 & 2 & 2 & 5 & 3 & 5 & 4 & 3 & 6 & 7 & 1 & 6 & 2 & 4 & 2 & 5 \\
\hline 8 & 1 & 1 & 1 & 2 & 4 & 9 & 1 & 1 & 6 & 4 & 3 & 2 & 7 & 1 & 4 & 3 & 3 & 3 & 1 & 2 \\
\hline 9 & 1 & 1 & 4 & 7 & 5 & 1 & 1 & 1 & 7 & 3 & 8 & 3 & 7 & 3 & 1 & 2 & 5 & 2 & 1 & 4 \\
\hline 10 & 1 & 4 & 1 & 2 & 2 & 1 & 1 & 5 & 2 & 2 & 2 & 5 & 1 & 1 & 1 & 1 & 2 & 3 & 3 & 5 \\
\hline Arc & & & & & & & & & & & & & & & & & & 12) & & \\
\hline $\mathrm{b}, \mathrm{c}$ & b & c & b & c & b & c & b & c & b & c & b & c & b & c & b & c & b & c & b & c \\
\hline 0 & 6 & 2 & 5 & 6 & 3 & 5 & 4 & 9 & 6 & 4 & 7 & 7 & 1 & 4 & 1 & 4 & 3 & 3 & 2 & 3 \\
\hline 1 & 2 & 8 & 3 & 6 & 2 & 6 & 2 & 8 & 1 & 3 & 2 & 6 & 1 & 3 & 1 & 4 & 3 & 6 & 2 & 5 \\
\hline 2 & 2 & 7 & 1 & 5 & 3 & 5 & 3 & 7 & 1 & 3 & 2 & 5 & 1 & 3 & 2 & 5 & 2 & 8 & 3 & 8 \\
\hline 3 & 3 & 6 & 2 & 5 & 1 & 7 & 1 & 6 & 2 & 2 & 1 & 4 & 2 & 4 & 3 & 6 & 2 & 7 & 4 & 6 \\
\hline 4 & 2 & 2 & 2 & 4 & 1 & 6 & 2 & 5 & 2 & 1 & 1 & 3 & 1 & 5 & 4 & 2 & 1 & 4 & 2 & 7 \\
\hline 5 & 2 & 5 & 2 & 4 & 1 & 5 & 3 & 4 & 1 & 5 & 3 & 6 & 1 & 5 & 3 & 4 & 1 & 9 & 1 & 3 \\
\hline 6 & 1 & 4 & 1 & 3 & 2 & 3 & 4 & 4 & 3 & 5 & 2 & 5 & 2 & 5 & 2 & 7 & 1 & 4 & 1 & 5 \\
\hline 7 & 2 & 4 & 1 & 7 & 3 & 4 & 5 & 3 & 1 & 6 & 1 & 6 & 1 & 6 & 1 & 6 & 4 & 5 & 1 & 4 \\
\hline 8 & 1 & 2 & 3 & 6 & 6 & 4 & 8 & 2 & 2 & 4 & 3 & 7 & 3 & 6 & 4 & 3 & 2 & 4 & 1 & 3 \\
\hline 9 & 4 & 1 & 4 & 4 & 7 & 7 & 2 & 1 & 4 & 6 & 5 & 8 & 4 & 6 & 1 & 2 & 6 & 3 & 1 & 3 \\
\hline 10 & 1 & 3 & 2 & 3 & 1 & 2 & 1 & 4 & 2 & 2 & 3 & 1 & 1 & 2 & 2 & 1 & 4 & 1 & 5 & 2 \\
\hline
\end{tabular}

Table 1: Information about Figure 1

\section{Robust time-varying shortest path}

In this section, robust time-varying shortest path with arbitrary waiting times (RTVSP-AWT) is presented. Then, a new algorithm for solving RT-VSP-AWT is proposed. The algorithm holds for all problem RT-VSP-ZWT and RT-VSP-BWT, too.

The T-VSP formulation is given follows:

$$
\begin{gathered}
\min \sum_{(i, j) \in A} \sum_{t=0}^{T} c(i, j, t) f(i, j, t)+\sum_{i \in V} \sum_{t=0}^{T} c(i, t) f(i, t) \\
\text { s.t } \sum_{(s, i) \in A} \sum_{t=0}^{T} f(s, i, t)=1 \\
\sum_{j:(i, j) \in A, t^{\prime}+b\left(i, j, t^{\prime}\right)=t} f\left(i, j, t^{\prime}\right)+f(j, t)-\sum_{j:(j, i) \in A} f(j, i, t)=0 \quad \forall i \in V \backslash\{s, \rho\}, \forall t
\end{gathered}
$$




$$
\begin{aligned}
& \sum_{(i, \rho) \in A} \sum_{t=0}^{T} f(i, \rho, t)=1 \\
& 0 \leq f(i, j, t) \leq U(i, j, t) \\
& 0 \leq f(i, t) \leq U(i, t)
\end{aligned}
$$

where, $f(i, j, t)$ is considered as the flow travelling on the arc $(i, j)$ during the time $[t, t+b(i, j, t)]$ and $f(i, t)$ is defined as the waiting flow at vertex $i$ during time $[t, t+1]$. The above formulation is derived from a general model for a time-varying minimum cost problem where the time varying shortest path is obtained by sending one unit of flow from some source vertex $s$ to a sink node $\rho$. The sending and receiving of one unit of flow from $s$ to $\rho$ can be shown by constraints (2) and (4), respectively. The objective function is exhibited by constraint (1) such that the cost of the shortest path is attained by minimization of the objective function. The constraints shown in equality (3) are called balance constraints. The inequality constraints in (5) and (6) are referred to as arcs flow bound constraints and flow bound constraints of the vertices, respectively. Let $F$ be the set of feasible solutions of the above problem formulation. Consider that the arc costs for time $\mathrm{t}$ on arc $(i, j)$ take the value in $[c(i, j, t), c(i, j, t)+d(i, j, t)]$ such that $\forall(i, j) \in A, \forall t=0, \ldots, T: d(i, j, t) \geq 0$. Let $J:=\{(i, j, t): d(i, j, t)>0\}$ and $S \subseteq J$, the robust time-varying shortest path is:

$$
\begin{aligned}
& \min \sum_{(i, j) \in A} \sum_{t=0}^{T} c(i, j, t) f(i, j, t)+\sum_{i \in V} \sum_{t=0}^{T} c(i, t) f(i, t)+\max _{|S| \leq \Gamma} \sum_{(i, j, t) \in S} d(i, j, t) f(i, j, t) \\
& \text { s.t } \quad f \in F
\end{aligned}
$$

where $\Gamma \in[0,|A| T]$. Problem (7) can be rewritten as follows:

$$
\begin{aligned}
& \min _{f \in F} \sum_{(i, j) \in A} \sum_{t=0}^{T} c(i, j, t) f(i, j, t)+\sum_{i \in V} \sum_{t=0}^{T} c(i, t) f(i, t) \\
&+\max \sum_{(i, j, t) \in J} d(i, j, t) f(i, j, t) v(i, j, t) \\
& \text { s.t } \quad \sum_{(i, j, t) \in J} v(i, j, t) \leq \Gamma, \quad 0 \leq v(i, j, t) \leq 1 \quad \forall(i, j, t) \in J
\end{aligned}
$$

For fixed $f \in F$, by applying a strong duality theorem for the dual of the inner maximization problem of problem (8), we can rewrite problem (8) as follows: 


$$
\begin{aligned}
& \min _{f \in F} \sum_{(i, j) \in A} \sum_{t=0}^{T} c(i, j, t) f(i, j, t)+\min _{\theta, y}\left(\Gamma \theta+\sum_{(i, j, t) \in J} y(i, j, t)\right)+\sum_{i \in V} \sum_{t=0}^{T} c(i, t) f(i, t) \\
& \begin{array}{ll}
\text { s.t } y(i, j, t)+\theta \geq d(i, j, t) f(i, j, t) \quad \forall(i, j, t) \in J \\
y(i, j, t) \geq 0 \quad \forall(i, j, t) \in J
\end{array}
\end{aligned}
$$

Problem (9) is equivalent to the following problem:

$$
\min _{\theta \geq 0} Z(\theta)
$$

where

$$
Z(\theta)=\Gamma \theta+\min _{f, y}\left(\sum_{i \in V} \sum_{t=0}^{T} c(i, t) f(i, t)+\sum_{(i, j) \in A} \sum_{t=0}^{T} c(i, j, t) f(i, j, t)+\sum_{(i, j, t) \in J} y(i, j, t)\right)
$$

such that

$$
\begin{array}{ll}
y(i, j, t) \geq d(i, j, t) f(i, j, t)-\theta & \forall(i, j, t) \in J \\
y(i, j, t) \geq 0 & \forall(i, j, t) \in J \\
\theta \geq 0 & \\
f \in F &
\end{array}
$$

By eliminating the variables $y(i, j, t)$ in equation (11) and for fixed $\theta \geq 0, Z(\theta)$ is equal to:

$$
\begin{aligned}
\min _{f \in F}\left(\sum_{i \in V} \sum_{t=0}^{T} c(i, t) f(i, t)\right. & +\sum_{(i, j) \in A} \sum_{t=0}^{T} c(i, j, t) f(i, j, t) \\
& \left.+\sum_{(i, j, t) \in J} d(i, j, t) \max \left\{f(i, j, t)-\frac{\theta}{d(i, j, t)}, 0\right\}\right)
\end{aligned}
$$

Theorem 2. For fixed $\theta \geq 0$, problem (12), can be solved as a T-VSP.

Proof. For every $\operatorname{arc}(i, j)$ with $\operatorname{arc} d(i, j, t)>0$, we introduce two new nodes $i^{\prime}$ and $j^{\prime}$ and delete original arc $(i, j)$, then insert four new $\operatorname{arcs}\left(i, i^{\prime}\right),\left(i^{\prime}, j^{\prime}\right),\left(j^{\prime}, j\right)$ and $\left(i^{\prime}, j\right)$. The costs, capacities and transit times are sets as follow:

$$
\begin{array}{llr}
c\left(i, i^{\prime}, t\right):=c(i, j, t), & U\left(i, i^{\prime}, t\right):=U(i, j, t), & b\left(i, i^{\prime}, t\right):=b(i, j, t), \\
c\left(i^{\prime}, j^{\prime}, t\right):=d(i, j, t), & U\left(i^{\prime}, j^{\prime}, t\right):=\infty, & b\left(i^{\prime}, j^{\prime}, t\right):=0, \\
c\left(j^{\prime}, j, t\right):=0, & U\left(j^{\prime}, j, t\right):=\infty, & b\left(j^{\prime}, j, t\right):=0, \\
c\left(i^{\prime}, j, t\right):=0, & U\left(i^{\prime}, j, t\right):=\frac{\theta}{d(i, j, t)}, & b\left(i^{\prime}, j, t\right):=0 .
\end{array}
$$


The solution to problem (12) is obtained by solving the T-VSP problem and implementing the changes as mentioned before. If $f(i, j, t) \leq \frac{\theta}{d(i, j, t)}$, the flow is routed along the $\operatorname{arcs}\left(i, i^{\prime}\right)$ and $\left(i^{\prime}, j\right)$. Therefore:

$$
c\left(i, i^{\prime}, t\right) f(i, j, t)+c\left(i^{\prime}, j, t\right) f(i, j, t)=c(i, j, t) f(i, j, t) .
$$

If $f(i, j, t) \geq \frac{\theta}{d(i, j, t)}$, first the flow is routed the arc $\left(\mathrm{i}, \mathrm{i}^{\prime}\right)$, then an amount of $\frac{\theta}{d(i, j, t)}$ is routed along the arc $\left(i^{\prime}, j\right)$ and the excess amount $f(i, j, t)-\frac{\theta}{d(i, j, t)}$ is routed along the arc $\left(i^{\prime}, j^{\prime}\right)$ and $\left(j^{\prime}, j\right)$. Therefore, the arising cost is:

$$
\begin{aligned}
& c\left(i, i^{\prime}, t\right) f(i, j, t)+c\left(i^{\prime}, j, t\right) \frac{\theta}{d(i, j, t)}+c\left(i^{\prime}, j^{\prime}, t\right)\left(f(i, j, t)-\frac{\theta}{d(i, j, t)}\right)+c\left(j^{\prime}, j, t\right) \\
& \left(f(i, j, t)-\frac{\theta}{d(i, j, t)}\right)=c(i, j, t) f(i, j, t)+d(i, j, t)\left(f(i, j, t)-\frac{\theta}{d(i, j, t)}\right) .
\end{aligned}
$$

In both cases, the transit time from $i$ to $j$ is equal to $b(i, j, t)$ and the cost of objective function is the same as the cost of the robust problem.

Theorem 3. $Z(\theta)$ is a convex function of $\theta$.

Proof. Let $\left(f_{1}, y_{1}\right)$ and $\left(f_{2}, y_{2}\right)$ be the optimal solutions of problem (11) corresponding to $\theta_{1}$ and $\theta_{2}$, respectively. The feasible region of problem is convex and the following holds:

$$
\lambda Z\left(\theta_{1}\right)+(1-\lambda) Z\left(\theta_{2}\right) \geq Z\left(\lambda \theta_{1}+(1-\lambda) \theta_{2}\right)
$$

\section{Algorithm RT-VSP-AWT}

Using the results of above theorems and one-dimensional minimization, we propose the following algorithm to find the optimal solution $\theta^{*}$ of problem 10 .

\section{Algorithm RT-VSP-AWT:}

1- Begin

2- Let robust time-varying shortest path (RT-VSP) by the $G(V, A, b, c, U)$.

3- Let $\Gamma \in[0, \bar{\Gamma}]$, where $\bar{\Gamma}:=|(i, j, t): d(i, j, t)>0|$.

4- Set $\bar{\theta}=\max _{(i, j) \in A, t \in T} U(i, j, t) d(i, j, t)$ and $\theta_{1}:=0$.

5- Give a $\epsilon$ as convergence criterion.

6- Calculate $Z\left(\frac{\bar{\theta}}{3}\right)$ and $Z\left(\frac{2 \bar{\theta}}{3}\right)$. 
7- Let $L:=\bar{\theta}-\theta_{1}$ and $L>\epsilon$. Do:

If

$$
Z\left(\frac{\bar{\theta}}{3}\right)<Z\left(\frac{2 \bar{\theta}}{3}\right)
$$

do $\bar{\theta}:=\frac{2 \bar{\theta}}{3}$ and go to step 6 .

If

$$
Z\left(\frac{\bar{\theta}}{3}\right)>Z\left(\frac{2 \bar{\theta}}{3}\right)
$$

do $\theta_{1}:=\frac{\bar{\theta}}{3}$ and go to step 6 .

If

$$
Z\left(\frac{\bar{\theta}}{3}\right)=Z\left(\frac{2 \bar{\theta}}{3}\right)
$$

do $\theta_{1}:=\frac{\bar{\theta}}{3}, \bar{\theta}:=\frac{2 \bar{\theta}}{3}$ and go to step 6 .

8- Let $\theta^{*}:=\frac{\bar{\theta}+\theta_{1}}{2}$ and evaluate $Z\left(\theta^{*}\right)$ as a optimal point.

9- End.

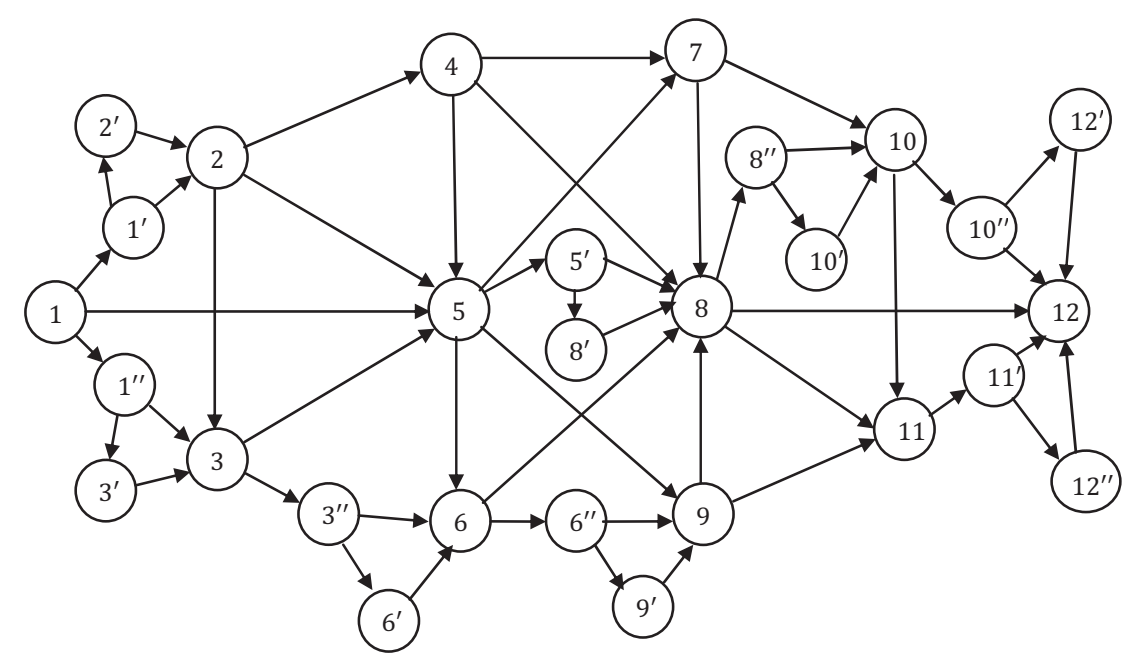

Figure 2: Result of the Theorem 2 in Example 2

Example 2. Consider the given network in Figure 1. Assume that transit times and transit costs for arcs are the same as for Example 1 except for the following arcs:

$$
(2,4),(2,5),(4,7),(6,8),(7,10),(8,12) \text { and }(9,11) .
$$

For $\operatorname{arcs}(2,4),(2,5),(4,7)$ and $(6,8)$, let:

$$
(b(i, j, t), c(i, j, t))=(2,1) \quad \forall t \in T=\{0,1, \ldots, 10\} .
$$


For arc $(7,10),(8,12)$ and $(9,11)$, let:

$$
(b(i, j, t), c(i, j, t))=(3,2) \quad \forall t \in T=\{0,1, \ldots, 10\}
$$

and

$$
U(i, j, t)=1 \quad \forall(i, j) \in A, \quad \forall t \in T=\{0,1, \ldots, 10\} .
$$

Moreover, information on the arcs $(1,2),(1,3),(3,6),(6,9),(5,8),(8,10),(10,12)$ and $(11,12)$ are given in Table 2. The problem is finding the cost of the robust shortest

\begin{tabular}{|c|c|c|c|c|c|c|c|c|}
\hline Arc & \multicolumn{2}{|c|}{$(1,2)$} & \multicolumn{2}{|c|}{$(1,3)$} & \multicolumn{2}{|c|}{$(3,6)$} & \multicolumn{2}{|c|}{$(5,8)$} \\
\hline b,c & b & $\mathbf{c}$ & b & c & b & c & b & c \\
\hline 0 & 1 & {$[2,10]$} & 2 & {$[3,12]$} & 1 & {$[1,11]$} & 1 & {$[2,12]$} \\
\hline 1 & 1 & {$[3,11]$} & 1 & {$[3,13]$} & 2 & {$[2,13]$} & 1 & {$[3,12]$} \\
\hline 2 & 2 & {$[4,13]$} & 1 & {$[4,15]$} & 1 & {$[1,13]$} & 2 & {$[4,12]$} \\
\hline 3 & 1 & {$[5,15]$} & 1 & {$[5,16]$} & 3 & {$[4,17]$} & 1 & {$[5,15]$} \\
\hline 4 & 3 & {$[2,9]$} & 2 & {$[4,13]$} & 1 & {$[5,15]$} & 3 & {$[2,11]$} \\
\hline 5 & 2 & {$[3,11]$} & 2 & {$[6,14]$} & 2 & {$[6,15]$} & 2 & {$[3,11]$} \\
\hline 6 & 1 & {$[4,12]$} & 1 & {$[1,10]$} & 1 & {$[8,16]$} & 1 & {$[4,15]$} \\
\hline 7 & 2 & {$[5,10]$} & 3 & {$[3,16]$} & 1 & {$[2,12]$} & 2 & {$[5,17]$} \\
\hline 8 & 1 & {$[1,16]$} & 1 & {$[2,16]$} & 4 & {$[9,19]$} & 1 & {$[1,18]$} \\
\hline 9 & 1 & {$[1,14]$} & 4 & {$[7,18]$} & 5 & {$[1,17]$} & 1 & {$[1,19]$} \\
\hline 10 & 1 & {$[4,12]$} & 1 & {$[2,11]$} & 2 & {$[1,14]$} & 1 & {$[5,15]$} \\
\hline Arc & \multicolumn{2}{|c|}{$(6,9)$} & \multicolumn{2}{|c|}{$(8,10)$} & \multicolumn{2}{|c|}{$(10,12)$} & \multicolumn{2}{|c|}{$(11,12)$} \\
\hline b,c & b & c & b & & b & & $\mathbf{b}$ & $\mathrm{c}$ \\
\hline 0 & 6 & {$[2,10]$} & 5 & {$[6,13]$} & 3 & {$[5,17]$} & 4 & {$[9,20]$} \\
\hline 1 & 2 & {$[8,16]$} & 3 & {$[6,13]$} & 2 & {$[6,18]$} & 2 & {$[8,19]$} \\
\hline 2 & 2 & {$[7,15]$} & 1 & {$[5,12]$} & 3 & {$[5,17]$} & 3 & {$[7,18]$} \\
\hline 3 & 3 & {$[6,14]$} & 2 & {$[5,12]$} & 1 & {$[7,19]$} & 1 & {$[6,17]$} \\
\hline 4 & 2 & {$[2,10]$} & 2 & {$[4,11]$} & 1 & {$[6,18]$} & 2 & {$[5,16]$} \\
\hline 5 & 2 & {$[5,13]$} & 2 & {$[4,11]$} & 1 & {$[5,17]$} & 3 & {$[4,15]$} \\
\hline 6 & 1 & {$[4,12]$} & 1 & {$[3,10]$} & 2 & {$[3,15]$} & 4 & {$[4,15]$} \\
\hline 7 & 2 & {$[4,12]$} & 1 & {$[7,14]$} & 3 & {$[4,16]$} & 5 & {$[3,14]$} \\
\hline 8 & 1 & {$[2,10]$} & 3 & {$[6,13]$} & 6 & {$[4,16]$} & 8 & {$[2,13]$} \\
\hline 9 & 4 & {$[1,9]$} & 4 & {$[4,11]$} & 7 & {$[7,19]$} & 2 & {$[1,12]$} \\
\hline 10 & 1 & {$[3,11]$} & 2 & {$[3,10]$} & 1 & {$[2,14]$} & 1 & {$[4,15]$} \\
\hline
\end{tabular}
path from vertex 1 to vertex 12 .

Table 2: Information about Figure 1 for Example 2

The transit costs for arcs $(1,2),(1,3),(3,6),(6,9),(5,8),(8,10),(10,12)$ and $(11,12)$ are uncertain. By using of the Theorem 2, we have Figure 2 and Table 3. Let $\varepsilon=1$. Using the RT-VSP-AWT algorithm, the robust time-varying shortest paths from vertex 1 to vertex 12 are obtained as 5.35, 5.70, 6.05 and 6.40 for $\Gamma=1,2,3$ and 4 , respectively. 


\begin{tabular}{|c|c|c|c|c|c|c|c|c|c|c|c|c|c|c|c|c|}
\hline Arcs & \multicolumn{2}{|c|}{$\left(1,1^{\prime}\right)$} & \multicolumn{2}{|c|}{$\left(1^{\prime}, 2^{\prime}\right)$} & \multicolumn{2}{|c|}{$\left(1,1^{\prime \prime}\right)$} & \multicolumn{2}{|c|}{$\left(1^{\prime \prime}, 3^{\prime}\right)$} & \multicolumn{2}{|c|}{$\left(3,3^{\prime \prime}\right)$} & \multicolumn{2}{|c|}{$\left(3^{\prime \prime}, 6^{\prime}\right)$} & \multicolumn{2}{|c|}{$\left(5,5^{\prime}\right)$} & \multicolumn{2}{|c|}{$\left(5^{\prime}, 8^{\prime}\right)$} \\
\hline$b, c$ & $\mathrm{~b}$ & c & $b$ & c & $b$ & c & $b$ & c & $\mathrm{b}$ & c & b & c & $\mathrm{b}$ & $\mathrm{c}$ & b & c \\
\hline 0 & 1 & 2 & 0 & 8 & 2 & 3 & 0 & 9 & 1 & 1 & 0 & 10 & 1 & 2 & 0 & 10 \\
\hline 1 & 1 & 3 & 0 & 8 & 1 & 3 & 0 & 10 & 2 & 2 & 0 & 11 & 1 & 3 & 0 & 9 \\
\hline 2 & 2 & 4 & 0 & 9 & 1 & 4 & 0 & 11 & 1 & 1 & 0 & 12 & 2 & 4 & 0 & 8 \\
\hline 3 & 1 & 5 & 0 & 10 & 1 & 5 & 0 & 12 & 3 & 4 & 0 & 13 & 1 & 5 & 0 & 10 \\
\hline 4 & 3 & 2 & 0 & 7 & 2 & 4 & 0 & 9 & 1 & 5 & 0 & 10 & 3 & 2 & 0 & 9 \\
\hline 5 & 2 & 3 & 0 & 8 & 2 & 6 & 0 & 8 & 2 & 6 & 0 & 9 & 2 & 3 & 0 & 8 \\
\hline 6 & 1 & 4 & 0 & 8 & 1 & 1 & 0 & 9 & 1 & 8 & 0 & 8 & 1 & 4 & 0 & 11 \\
\hline 7 & 2 & 5 & 0 & 5 & 3 & 3 & 0 & 13 & 1 & 2 & 0 & 10 & 2 & 5 & 0 & 12 \\
\hline 8 & 1 & 1 & 0 & 15 & 1 & 2 & 0 & 14 & 4 & 9 & 0 & 10 & 1 & 1 & 0 & 17 \\
\hline 9 & 1 & 1 & 0 & 13 & 4 & 7 & 0 & 11 & 5 & 1 & 0 & 16 & 1 & 1 & 0 & 18 \\
\hline 10 & 1 & 4 & 0 & 8 & 1 & 2 & 0 & 9 & 2 & 1 & 0 & 13 & 1 & 5 & 0 & 10 \\
\hline $\operatorname{arcs}$ & \multicolumn{2}{|c|}{$\left(6,6^{\prime \prime}\right)$} & \multicolumn{2}{|c|}{$\left(6^{\prime \prime}, 9\right)$} & \multicolumn{2}{|c|}{$\left(8,8^{\prime \prime}\right)$} & \multicolumn{2}{|c|}{$\left(8^{\prime \prime}, 10^{\prime}\right)$} & \multicolumn{2}{|c|}{$\left(10,10^{\prime \prime}\right)$} & \multicolumn{2}{|c|}{$\left(10^{\prime \prime}, 12^{\prime}\right)$} & \multicolumn{2}{|c|}{$\left(11,11^{\prime}\right)$} & \multicolumn{2}{|c|}{$\left(11^{\prime}, 12^{\prime \prime}\right)$} \\
\hline$b, c$ & b & c & $b$ & c & $\mathrm{b}$ & c & $b$ & c & b & c & $b$ & c & $b$ & c & b & c \\
\hline 0 & 6 & 2 & 0 & 8 & 5 & 6 & 0 & 13 & 3 & 5 & 0 & 12 & 4 & 9 & 0 & 11 \\
\hline 1 & 2 & 8 & 0 & 8 & 3 & 6 & 0 & 13 & 2 & 6 & 0 & 12 & 2 & 8 & 0 & 11 \\
\hline 2 & 2 & 7 & 0 & 8 & 1 & 5 & 0 & 13 & 3 & 5 & 0 & 12 & 3 & 7 & 0 & 11 \\
\hline 3 & 3 & 6 & 0 & 8 & 2 & 5 & 0 & 13 & 1 & 7 & 0 & 12 & 1 & 6 & 0 & 11 \\
\hline 4 & 2 & 2 & 0 & 8 & 2 & 4 & 0 & 13 & 1 & 6 & 0 & 12 & 2 & 5 & 0 & 11 \\
\hline 5 & 2 & 5 & 0 & 8 & 2 & 4 & 0 & 13 & 1 & 5 & 0 & 12 & 3 & 4 & 0 & 11 \\
\hline 6 & 1 & 4 & 0 & 8 & 1 & 3 & 0 & 13 & 2 & 3 & 0 & 12 & 4 & 4 & 0 & 11 \\
\hline 7 & 2 & 4 & 0 & 8 & 1 & 7 & 0 & 13 & 3 & 4 & 0 & 12 & 5 & 3 & 0 & 11 \\
\hline 8 & 1 & 2 & 0 & 8 & 3 & 6 & 0 & 13 & 6 & 4 & 0 & 12 & 8 & 2 & 0 & 11 \\
\hline 9 & 4 & 1 & 0 & 8 & 4 & 4 & 0 & 13 & 7 & 7 & 0 & 12 & 2 & 1 & 0 & 11 \\
\hline 10 & 1 & 3 & 0 & 8 & 2 & 3 & 0 & 13 & 1 & 2 & 0 & 12 & 1 & 4 & 0 & 11 \\
\hline
\end{tabular}

Table 3: Information about Figure 2

\section{Conclusion}

In this paper, we worked on the robust time-varying shortest path with arbitrary waiting times at vertices. We concentrated on the problem in which arc costs are uncertain and belong to intervals. Our model finds a path between the source vertex and the sink vertex with the minimum total costs in which transit and waiting costs are included. A robust formulation was proposed to take care of uncertainty, plus an algorithm was developed to clarify steps of finding the solution.

\section{References}

[1] Ahuja, R.K., Magnanti, T.L. and Orlin, J.B. (1993). Network Flows: Theory, Algorithms, and Applications. DC: Prentice-Hall, Englewood Cliffs.

[2] Aronson, j. (1989). A survey of dynamic network flows. ANN. OPER. RES. 20, 1-66.

[3] Bertsimas, D., and Sim, M. (2003). Robust discrete optimization and network flows. Math. Program. B. 98, 49-71.

[4] Cai, X., Kloks, T., and Wong, C.K. (1997). Time-varying shortest path problems with constraints. Networks 29, 141-149.

[5] Cai, X., Sha, D., and Wong, C.K. (2001). Time-varying minimum cost flow problems. EUR. J. OPER. RES. 131, 352-374. 
[6] Cai, X., Sha, D., and Wong, C.K. (2007). Time-Varying Network Optimization. DC: Springer Science+Business Media, New York.

[7] Chabini,L. (1998). Discrete dynamic shortest path problems in transportation applications: Complexity and algorithms with optimal run time. TRANSPORT. RES. REC. 1645, 170-175.

[8] Fonoberova, M. (2007). Optimal Flows in Dynamic Networks and Algorithm for their Finding, Ph.D. Thesis, Moldova University.

[9] Ford, L.R., and Fulkerson, D.R. (1958). Constructing maximal dynamic flows from static flows. OPER. RES. 6, 419-433.

[10] Glockner, G., and Nemhauser, G. (2000). A dynamic network flow problem with uncertain arc capacities: formulation and problem structure. OPER. RES. 48, 33-242.

[11] Hashemi, M., and Nasrabadi, E. (2012). Solving continuous-time dynamic network flows. J. Glob. Optim. 53, 497-524.

[12] Hoppe, B., (1995). Efficient Dynamic Network Flow Algorithms. Ph.D. Thesis, Cornell University.

[13] Orda, A., and Rom, R. (1990). Shortest-path and minimum-delay algorithms in networks with time-dependent edge length. J. ASSOC. COMPUT. MACH. 37, 607-625.

[14] Orda, A., and Rom, R. (1991). Minimum weight paths in time-dependent networks. Networks. 21 295-320.

[15] Nasrabadi, E., and Hashemi, M. (2010). Minimum cost time-varying network flow problems. OPTIM. METHOD. SOFTW. 25(3), 429-447.

[16] Salehi Fathabadi, H., and Hosseini, S.A. (2010). Maximum flow problem on dynamic generateve network flows with time-varying bounds. APPL. MATH. MODEL. 34, 2136-2147.

[17] Shirdel, GH., and Rezapour, H. (2015). K-Objective Time-Varying Shortest Path Problem with Zero Waiting Times at Vertices. Trends. Appl. Sci. Res. 10, 278-285.

[18] Shirdel, GH., and Rezapour, H. (2016). Time-Varying Shortest Path With triangular Fuzzy Costs. Advances and Applications in Mathematical Sciences. 15(2), 75-85. 